\title{
Sabin and wild type polioviruses from children who presented with acute flaccid paralysis in Nigeria
}

\author{
Adedeji $\mathrm{AO}^{1}$, *Okonko $\mathrm{IO}^{2}, \mathrm{Adu} \mathrm{FD}^{3}$ \\ 1. Department of Veterinary Microbiology \& Parasitology, Faculty of Veterinary Medicine, University of Ibadan, Ibadan, \\ Nigeria \\ 2. Medical Microbiology \& Virology Unit, Department of Microbiology, University of Port Harcourt, East-West Road, \\ P.M.B. 5323, Choba, Port Harcourt, Rivers State, Nigeria; \\ 3.Department of Virology, College of Medicine, University of Ibadan, Ibadan, Nigeria
}

\begin{abstract}
Background: Sensitive poliovirus surveillance to detect vaccine-derived-polioviruses will continue to increase in importance. Objective: Isolating and identifying poliovirus strains from children of pediatrics age in Nigeria.

Methods: A total of 120 fecal samples were randomly collected from children under the age of five who presented with acute flaccid paralysis. Samples were tested by tissue culture technique and further characterized by intratypic differentiation testing using ELISA and PCR methods.

Results: The study confirmed the presence of 22(18.3\%) enteroviral isolates comprising 19(86.4\%) polioviruses and $3(13.6 \%)$ non-polio enteroviruses. These 19 polioviruses include: Sabin-type poliovirus-1 (15.8\%), poliovirus-2 (10.5\%), poliovirus-3 (10.5\%) and wild-type poliovirus-1 (63.2\%) isolates. It showed that poliovirus infection was higher in children ages 6-11 months (18.9\%), females (18.4\%), northern states $(91.0 \%)$ with no vaccination record (75.0\%). Wild-type poliovirus1 was isolated from the stool samples of 12(54.6\%) children from northern states and in all age groups except 18-23 months. No significant differences $(\mathrm{P}>0.05)$ between poliovirus infection and age $(18.9 \%$ vs. $17.7 \% ; 81.9 \%$ vs. $18.2 \%)$ and sex $(18.3 \%$ vs. $18.4 \%)$. There was significant differences $(\mathrm{P}<0.05)$ between poliovirus infection and location $(91.0 \%$ vs. $9.0 \%$ ) and history of polio vaccination $(75.0 \%$ vs. $0.0 \%)$. No wild-type poliovirus was found in those with complete vaccination.

Conclusion: This study further confirms the presence of Sabin and wild-type poliovirus among children in Nigeria. The isolation of Sabin strain of poliovirus is advantageous to the polio eradication program as it is capable of inducing natural immunity in susceptible hosts. Transmission of wild-type poliovirus among children with incomplete vaccination poses a serious threat to polio eradication program in Nigeria. Environmental and serological surveillance with larger sample size are important for monitoring poliovirus circulation in Nigeria.
\end{abstract}

Keywords: Acute flaccid paralysis, ELISA, ITD, RT-PCR, Poliovirus, Sabin

African Health Sciences 2012; 12(3): 345 - 354 http://dx.doi.org/10.4314/ahs.v12i3.16

\section{Introduction}

Poliomyelitis, an acute viral disease of the central nervous system of humans, can be effectively controlled and eliminated through the use of inactivated polio vaccine (IPV) or live attenuated oral

\begin{tabular}{|l|}
\hline *Correspondence author: \\
Iheanyi O. Okonko \\
Medical Microbiology Unit \\
Department of Microbiology \\
University of Port Harcourt \\
P.M.B. 5323, Choba, East-West Road \\
Port Harcourt \\
Rivers State, Nigeria \\
Tel: +2348035380891 \\
E - M a i 1: m a c 2 f i n n e y @ y a h o o.c o m \\
iheanyi.okonko@uniport.edu.ng \\
\hline
\end{tabular}

African Health Sciences Vol 12 No 3 September 2012 polio vaccine (OPV). Therefore, the goal of the Global Polio Eradication Initiative (GPEI) is to stop the global transmission of poliovirus1 via use of the vaccines. Poliomyelitis, which occurs worldwide, is basically caused by three serotypes of wild poliovirus (i.e. types 1, 2, and 3), although vaccineassociated paralytic poliomyelitis (VAPP) have been documented among oral polio vaccine recipients that excreted vaccine-derived poliovirus ${ }^{1-4}$. The global eradication of wild type 2 poliovirus (WPV) in 1999 was a remarkable achievement ${ }^{5}$ however, between 2005 to 2009 the vaccine-induced population immunity to type 2 vaccine derived poliovirus (VDPV) declined significantly in all the geographical zones in Nigeria (4.7\% to $8.8 \%$ annually) ${ }^{5,6}$ 
The central strategy of the World Health Organization global polio eradication initiatives (GPEI) is widespread use of oral polio vaccine at high rates of coverage ${ }^{4}$. This strategy has reduced the global incidence of polio by over $99 \%$ since the start of the Initiative in 1988 and has restricted wild poliovirus circulation to countries in western and central Africa and southern Asia ${ }^{4,7}$ Though outbreaks of infection caused by circulating vaccine-derived poliovirus (cVDPV) can be prevented by maintenance of high rates of oral polio vaccine coverage, this is bedevilled by the occurrence of vaccine-associated paralytic poliomyelitis (VAPP) which is associated with the inherent genetic instability of the live attenuated oral polio vaccine strains $s^{4,8}$

Currently, endemic infections with wild polio virus are limited to three countries where transmission has not been interrupted (these are Nigeria, Afghanistan and Pakistan); and to several other countries due to importation of wild poliovirus.9-11 The number of reported cases of wild poliovirus infection dropped from 350,000 in 1988 to 1,596 in 2009 (with the apparent elimination of poliovirus type 2 in 1999).10,12-13 In countries where poliovirus infection is still endemic, paralytic disease is most often caused by poliovirus type 1, less frequently by poliovirus type 3 , and least frequently by poliovirus type 2.14 The virus is transmitted from person to person primarily by direct fecal-oral contact. However, it also can be transmitted by indirect contact with infectious saliva or feces or by contaminated food or water ${ }^{14}$.

Despite significant success of the global polio eradication initiatives (GPEI) in Nigeria, Afghanistan, India, Pakistan, wild poliovirus still circulate due to persistently high proportions of under and unimmunized children.15. Mucosal immunity induced by oral polio vaccine is imperfect and potentially allows immunized individuals to participate in asymptomatic wild poliovirus transmission in settings with efficient fecal-oral transmission of infection ${ }^{16}$. Since 2005, a total of 355 acute flaccid paralysis (AFP) cases associated with an outbreak of cVDPV-2 have been reported in 11 northern and three central states of Nigeria, ${ }^{5,17-19}$. The outbreak peaked at 153 cases in 2009 , but 27 cases were detected in 2010, and five cases (representing three transmission chains) were detected through March 2011.18 Genetic analysis indicated that detected cases represent at least seven concurrent outbreaks arising from multiple circulating vaccine- derived polioviruses (cVDPV)-2 emergences during 2004-2006 ${ }^{17,19}$. The outbreak occurred in northern states, where coverage attained through routine vaccination with oral polio vaccine was low and oral polio vaccine supplementary immunization activities (SIAs) were infrequent ${ }^{5,17-19}$.

The largest recorded outbreak of the circulating vaccine-derived polioviruses, especially type 2, occurred in Nigeria in 2005-2009. This incidence provided a unique but rare opportunity to compare the potency of both the Sabin-like poliovirus type 2 and circulating vaccine-derived polioviruses (cVDPV)-2 for differential diagnosis purpose.6 The detection of numerous isolates with $<1 \%$ divergence that were ancestral to cVDPV2 lineages in Nigeria ${ }^{19}$ prompted a redefinition of vaccine-derived polioviruses-2 for reporting purposes by the Global Polio Laboratory Network. This has been redefined to include isolates with $>0.6 \%$ divergence. This was applied beginning in 2010.20 After the global eradication of wild polioviruses, the risk of paralytic poliomyelitis from polioviruses will still exist and require active management. Possible reintroductions of poliovirus that can spread rapidly in unprotected populations present challenges to policymakers $^{21}$.

An efficient acute flaccid paralysis (AFP) surveillance system supported by a WHO accredited laboratory network for the virological diagnosis of wild poliovirus infections is essential not only for the detection of cases of poliomyelitis but also for documentation of the success of poliovirus eradication efforts ${ }^{11}$. The aim of this study is therefore to isolate and identify wild-type and Sabinlike poliovirus strains from children under the age of five who presented with acute flaccid paralysis in Nigeria. It is envisaged that isolation and identification of wild-type and Sabin-like poliovirus strains from children under the age of five in Nigeria for continued transmission of wild poliovirus may provide supplementary information on the persistent transmission of wild poliovirus in the country.

\section{Methods}

\section{Study population and Sample collection}

A total of one hundred and twenty stool samples were collected from children who presented with acute flaccid paralysis in Nigeria from January to July, 2008. The children were stratified according to age, gender and locations. Stool samples were randomly picked from those brought by Disease Surveillance and Notification Officers (DSNOs) to 
the WHO National Reference laboratory for Polio, University College Hospital, Ibadan, Nigeria. The stool samples had been collected from children that presented with acute flaccid paralysis; these children were aged 0 to 59 months. The methods adopted for AFP surveillance and Laboratory testing of faecal specimens' isolation and typing of isolates, intratypic differentiation procedures were as described in the WHO Polio Laboratory Manual22 and the supplemental Manual of 200623 describing the New Algorithm Technique. In brief, surveillance officers, Disease Surveillance and Notification Officers (DSNOs) and Paediatricians in the following states (Zamfara, Kaduna, Niger, Sokoto, Abia, Delta, Benue, Federal Capital Territory [FCT], Kebbi, Kogi, Kwara and Nasarawa) collected two faecal specimens per AFP case, 24 hours apart and within 14 days of the onset of paralysis. These samples were sent in Giostyle box with ice packs to preserve the cold chain to the WHO National Polio/Intratypic Differentiation Laboratory, University College Hospital, Ibadan, Nigeria where they were stored at -20OC till tested.

Isolation and identification of poliovirus and
non-polio Enteroviruses (NPEVs) non-polio Enteroviruses (NPEVs)

The faecal specimen was processed according to standard protocols for virus isolation and characterization.22-24 These procedures identified the poliovirus and non-polio enteroviruses isolates into types 1,2 and 3.

\section{Cells}

Healthy monolayer of L20B and Rhabdomyosarcoma (RD) cell lines in maintenance medium (Eagle's MEM supplemented with 2\% fetal calf serum (FCS) were used in this study. The cells were seeded 48 hours earlier at a concentration of 105 cells per tubes in maintenance medium (Eagle's MEM supplemented with 2\% FCS).22-24 Sterile procedure was adhered to throughout the laboratory study.

\section{Processing of samples and isolation}

Feacal samples were pre-treated with chloroform before being inoculated on a healthy monolayer of L20B and RD cell lines in maintenance medium (Eagle's MEM supplemented with 2\% fetal calf serum (FCS). The cells were seeded 48 hours earlier into culture tubes with growth medium (Eagle's MEM supplemented with 10\% FCS). Each stool sample was inoculated into both L20B and RD cells.
The inoculated monolayers were observed daily for the characteristic enterovirus cytopathic effects of rounded, refractile cells detaching from the surface of the tube. The tubes with CPE up to $75 \%$ and above were harvested and kept at $-20^{\circ} \mathrm{C}$ to be passaged in fresh monolayer of the second cell lines to increase the titre, while those infected cells negative after 5 days of incubation were re-passaged on the same cell line; those found negative after another 5day incubation period were declared negative. The tubes positive for poliovirus-specific cytopathic effects were freeze-thawed thrice, spun at $4^{\circ} \mathrm{C}$ (specify the rpm and time) and the supernatant aliquoted and kept frozen at $-20^{\circ} \mathrm{C}$ as poliovirus isolates $^{22-24}$.

\section{Virus titration}

This was carried out as described by WHO manual22-24 and Oderinde et al.6 Sterile dilution tubes with screw caps were labelled 10-1 - 10-9 per virus isolate; $1.8 \mathrm{ml}$ of Maintenance medium (MM) was dispensed into the tubes. A volume of $0.2 \mathrm{ml}$ of the isolate was picked into the first tube (i.e. the 10-1) using a pipettor with sterile Aerosol Resistant Tips (ARTs), the tube was screwed cap and vortexed gently. With another pipette tip, $0.2 \mathrm{ml}$ from the first dilution tube was transferred to the second tube, the pipette tip was discarded; the tube was screwed cap and vortexed gently. This procedure was repeated until the last ten-fold serial virus dilution was made. A volume of 100 il of each virus dilutions was added to wells 1 to 10 per row of appropriately labelled 96 well microtitre plate. A volume of $100 i$ of maintenance medium was added to wells A11 to H12 of each row as cell controls. A volume of 100il each of L20B cells from a cell suspension containing $2 \times 105$ cells $/ \mathrm{ml}$ to all wells in rows $\mathrm{A}$ to $\mathrm{H}$ of the plate. The plate was covered with non-toxic sealer and incubated at $36^{\circ} \mathrm{C}$. The plate was examined daily for development of cytopathic effect, using an inverted microscope, and record daily readings for 5-7 days.

\section{Calculation of the virus titre using the Spearman $\&$ Karber formula}

This was carried out as described by WHO manual22-24. $\log$ CCID50 $=\mathrm{L}-\mathrm{d}(\mathrm{S}-0.5)$, where $: \mathrm{L}=\log$ of lowest dilution used in the test, $\mathrm{d}=$ difference between $\log$ dilutions steps, $\mathrm{S}=$ sum of proportion of 'positive' tests (i.e. cultures showing cytopathic effect). 
Intratypic differentiation of the Poliovirus (using serologic and molecular methods)

ELISA method for intratypic differentiation

Intratypic differentiation of the isolates, a test aimed at establishing whether poliovirus strain is wild-type or vaccine-derived, was carried out using an ELISA method as described by WHO manual22-24 and Adu et al.25

\section{PCR method for intratypic differentiation}

The Sabin genotype-specific RT-PCR was carried out as described by WHO manual.22-24 Viral RNA extraction and reverse transcriptase polymerase chain reaction (RT-PCR) were performed as previously described.22-24,25-27.

\section{Data analysis}

The prevalence for poliovirus was calculated by using children with positive samples as numerator and the total numbers of children enrolled in this study as denominator. The data generated from this study were presented using descriptive statistics. The data was subjected to Fisher's Exact Test for comparison of proportions to determine any significant relationship between infection rate, age, gender, location and history of polio vaccinations. Confidence level was set at $\mathrm{p}=0.05$.

\section{Results}

Virus isolation

Cytopathic effect was observed on the inoculated cells; the general cytopathic effect observed on L20B cells were changes in morphology, cell rounding and shrinking while cytopathic effect observed on RD cells were shrinking and complete lysis. From the 120 stool samples collected and inoculated into L20B and RD cell lines, 22(18.3\%) stool samples were positive for polio and non-polio enteroviruses (NPEVs). Of these 22 positive samples, 19 (86.4\%) showed poliovirus compatible cytopathic effect on L20B, as well as, on RD cells; 3 (13.6\%) out of the 22 samples showed cytopathic effect on RD only which while none $(0.0 \%)$ showed cytopathic effect on L20B only. The isolates obtained in RD cells only were non-polio enteroviruses.

Table 1: Distribution of Poliovirus strains in relation to ages of subjects

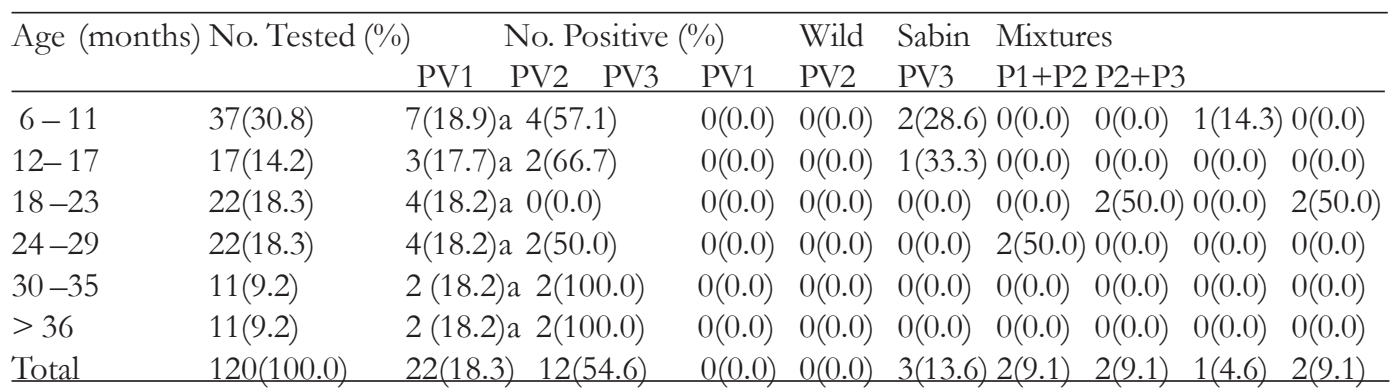

Mixtures $=$ Samples having $\mathrm{P} 1$ and $\mathrm{P} 2$ poliovirus strains and $\mathrm{P} 2$ and $\mathrm{P} 3$ poliovirus strains; $\mathrm{a}=$ Not Significant $(18.9 \%$ vs. $17.7 \% ; 18.9 \%$ vs. $18.2 \%$; P $>0.05)$

\section{Intratypic differentiation of the Poliovirus}

The use of ELISA and a Sabin genotype-specific RT-PCR confirmed the presence of 19 polioviruses, such as three Sabin-like poliovirus type-1 (15.8\%), two Sabin-like poliovirus type-2 (10.5\%), two Sabin poliovirus type-3 $(10.5 \%)$, and twelve wild poliovirus type-1 $(63.2 \%)$ isolates. It also confirmed the presence of three non-polio enteroviruses (13.6\%).

Table 2: Distribution of Polio virus isolates in relation to sex

\begin{tabular}{lll}
\hline Sex & No. Tested & No. Positive (\%) \\
\hline Male & $71(59.2)$ & $13(18.3) \mathrm{a}$ \\
Female & $49(40.8)$ & $9(18.4) \mathrm{a}$ \\
Total & $120(100.0)$ & $22(18.3)$ \\
\hline
\end{tabular}

$\mathrm{a}=$ Not Significant $(18.4 \%$ vs. $18.3 \%$; $\mathrm{P}>0.05)$

\section{Distribution of Poliovirus strains}

Distribution of poliovirus isolates in relation to the age of the children

Table 1 shows distribution poliovirus isolates in relation to the age of the children. The result indicates that more poliovirus isolates were obtained from the age groups 6 to 11 months of age, $7(18.9 \%)$ with four Wild poliovirus type-1 (57.1\%), two poliovirus-1 Sabin-like poliovirus isolates $(28.6 \%)$ and one $(14.3 \%)$ mixture of P1+P2. Sabin-like poliovirus type- 3 was only isolated from children in age group $18-23$ months of age $(50.0 \%)$ as shown in table 1. However, this difference was not statistically significant $(18.9 \%$ vs. $17.7 \% ; 81.9 \%$ vs. $18.2 \%, \mathrm{P}$ $>0.05$ ). 
Table 3: Distribution of isolates by location

\begin{tabular}{|c|c|c|c|c|c|}
\hline Location & No. $(\%)$ & Sabin (\%) & Wild (\%) & Mixture (\%) & Invalid (\%) \\
\hline \multicolumn{6}{|c|}{ Northern States } \\
\hline Benue & $0(0.0)$ & $0(0.0)$ & $0(0.0)$ & $0(0.0)$ & $0(0.0)$ \\
\hline FCT & $0(0.0)$ & $0(0.0)$ & $0(0.0)$ & $0(0.0)$ & $0(0.0)$ \\
\hline Kaduna & $4(18.2)$ & $0(0.0)$ & $2(50.0)$ & $2(50.0)$ & $0(0.0)$ \\
\hline Kebbi & $0(0.0)$ & $0(0.0)$ & $0(0.0)$ & $0(0.0)$ & $0(0.0)$ \\
\hline Kogi & $0(0.0)$ & $0(0.0)$ & $0(0.0)$ & $0(0.0)$ & $0(0.0)$ \\
\hline Kwara & $0(0.0)$ & $0(0.0)$ & $0(0.0)$ & $0(0.0)$ & $0(0.0)$ \\
\hline Nasarawa & $0(0.0)$ & $0(0.0)$ & $0(0.0)$ & $0(0.0)$ & $0(0.0)$ \\
\hline Niger & $4(18.2)$ & $4(100.0)$ & $0(0.0)$ & $0(0.0)$ & $0(0.0)$ \\
\hline Sokoto & $3(13.6)$ & $0(0.0)$ & $3(100.0)$ & $0(0.0)$ & $0(0.0)$ \\
\hline Zamfara & $9(40.9)$ & $0(0.0)$ & $7(77.8)$ & $0(0.0)$ & $2(22.2)$ \\
\hline Sub-total & $20(91.0) \mathrm{b}$ & $4(20.0)$ & $12(60.0)$ & $2(10.0)$ & $2(10.0)$ \\
\hline \multicolumn{6}{|c|}{ Southern States } \\
\hline Abia & $1(4.6)$ & $1(100.0)$ & $0(0.0)$ & $0(0.0)$ & $0(0.0)$ \\
\hline Delta & $1(4.6)$ & $0(0.0)$ & $0(0.0)$ & $1(100.0)$ & $0(0.0)$ \\
\hline Sub-total & $2(9.0) \mathrm{b}$ & $1(50.0)$ & $0(0.0)$ & $1(50.0)$ & $0(0.0)$ \\
\hline Overall tot & $122(18.3)$ & $5(22.7)$ & $12(54.6)$ & $3(13.6)$ & $2(9.1)$ \\
\hline
\end{tabular}

Invalid $=$ "double reactive" antigenic properties in the ELISA (i.e. reacting with both Sabin 2-specific and type 2 wild poliovirus-specific cross-absorbed rabbit antisera); $\mathrm{b}=$ Significant $(91.0 \%$ vs. $9.0 \%$; $\mathrm{P}<0.05)$

Distribution of poliovirus isolates in relation to sex of the children

Table 2 shows distribution of poliovirus serotypes in relation to sex of the children. This shows that $13 / 71(18.3 \%)$ of the poliovirus serotypes were obtained from male children while the remaining 9/49(18.4\%) were from the female children (table 3). This difference was not statistically significant (18.3\% vs. $18.4 \%, \mathrm{P}>0.05)$. Also 13 out of the 22 isolates (59.1\%) were from males while $9 / 22(40.9 \%)$ were from samples obtained from female children. This difference was statistically significant $(59.1 \%$ vs. $40.9 \%, \mathrm{P}<0.05)$.

\section{Distribution of poliovirus isolates in relation to locations of children}

Table 3 shows distribution of isolates by location. The highest number of the isolates was obtained from children in the northern states having 20(91.0\%), of which children in Zamfara State had 9(40.9\%), followed by Kaduna and Niger states having $4(18.2 \%)$ isolates respectively and Sokoto State $3(13.6 \%)$. While the remaining $2(9.0 \%)$ isolates were obtained from children in the southern states, $1(4.6 \%)$ from Abia State and 1(4.6\%) from Delta State respectively (table 3). No wild-type or Sabin-like poliovirus was isolated from the following states: Benue, FCT, Kebbi, Kogi, Kwara and Nasarawa.
This differences were statistically significant $(91.0 \%$ vs. $9.0 \%, \mathrm{P}<0.05)$.

Distribution of poliovirus isolates in relation to the polio vaccination status of the children Table 4 shows distribution of poliovirus in relation to the polio vaccination status of the children. Only five $(22.7 \%)$ Sabin- like polioviruses was isolated from children with complete vaccination (table 4). Three (25.0\%) Wild-type polioviruses were isolated from children with incomplete vaccination. A total of $9(75.0 \%)$ Wild-type polioviruses were isolated from children with no record of vaccination (table 4). This difference was statistically significant $(75.0 \%$ vs. $0.0 \% ; 75.0 \%$ vs. $25.0 \%, \mathrm{P}<0.05)$. 
Table 4: Distribution of poliovirus in relation to the vaccination status of the subjects

\begin{tabular}{lllll}
\hline Serotypes & $\begin{array}{l}\text { No. with complete } \\
\text { vaccination (\%) }\end{array}$ & $\begin{array}{l}\text { No. with incomplete } \\
\text { vaccination } \mathbf{( \% )}\end{array}$ & $\begin{array}{l}\text { No. not vaccinated } \\
\text { at all (\%) }\end{array}$ & Total (\%) \\
\hline PV1 Sabin & $3(100.0)$ & $0(0.0)$ & $0(0.0)$ & $3(13.6)$ \\
PV2 Sabin & $2(50.0)$ & $2(50.0)$ & $0(0.0)$ & $4(18.2)$ \\
PV3 Sabin & $0(0.0)$ & $0(0.0)$ & $0(0.0)$ & $0(0.0)$ \\
PV1 wild & $0(0.0) \mathrm{b}$ & $3(25.0) \mathrm{b}$ & $9(75.0) \mathrm{b}$ & $12(54.6)$ \\
PV2 wild $0(0.0)$ & $0(0.0)$ & $0(0.0)$ & $0(0.0)$ \\
PV3 wild $0(0.0)$ & $0(0.0)$ & $0(0.0)$ & $0(0.0)$ \\
Mixtures of 0(0.0) & $1(100.0)$ & $0(0.0)$ & $1(4.6)$ \\
Sabin 1 and 2 & & & \\
Mixtures of $0(0.0)$ & $2(100.0)$ & $0(0.0)$ & $2(9.1)$ \\
Sabin 2 and 3 & & & \\
Total & $5(22.7) \mathrm{b}$ & $8(36.4) \mathrm{b}$ & $9(40.9) \mathrm{b}$ & $22(100.0)$ \\
\hline
\end{tabular}

$\mathrm{b}=$ Significant $(75.0 \%$ vs. $0.0 \% ; 75.0 \%$ vs. $25.0 \% ; \mathrm{P}<0.05)$

\section{Discussion}

This study aimed at isolating and identifying poliovirus strains from children suspected of poliomyelitis in Nigeria. A total of 120 fecal samples were randomly collected from children under the age of five who presented with acute flaccid paralysis (AFP). Samples were tested by tissue culture technique and further characterized by intratypic differentiation testing using ELISA and PCR. Poliovirus 1 and poliovirus 2 Sabin poliovirus serotypes, wild-type poliovirus serotype 1 and non-polio enterovirus were found circulating in the study participants. The detection of wild poliovirus 1 serotype in this study is consistent with what was reported by Adu et al.25. Presently there is no vaccine for non-polio enteroviruses but as efforts are intensified on poliovirus eradication, there may be need to assess the impact of this group of viruses on the epidemiology of paralysis in children ${ }^{21}$.

In 2012, only three countries (Afghanistan, Nigeria and Pakistan) remain polio-endemic, down from more than 125 in $1988^{11}$. Persistent pockets of polio transmission in northern Nigeria and the border between Afghanistan and Pakistan are the current focus of the polio eradication initiative. As long as a single child remains infected, children in all countries are at risk of contracting polio ${ }^{111}$. For instance, in 2009-2010, 23 previously polio-free countries were re-infected due to imports of the virus. A report has shown that $95 \%$ of wild polioviruses are being transmitted in 8 high risk States (Kano, Jigawa, Borno, Zamfara, Sokoto, Kebbi, Yobe and Kasina) in Nigeria between 2011 and $2012^{11,28}$.
Wild type polioviruses serotype 1 and 3 on the other hand are still circulating in the major reservoirs in India and Nigeria ${ }^{29-30}$, although in this study, none of the isolates tested positive for poliovirus 3 (either wild or Sabin). According to Adu et $\mathrm{al}^{25}$, wild poliovirus type 1 has spread from reservoirs in northern Nigeria to 18 previously poliofree countries in 2002-2005, from Guinea in the west to Indonesia at the southeastern rim of Asia, resulting in over 1200 cases associated with imported virus since $2002^{31}$. Also, the use of restriction enzymes and a Sabin specific RT-PCR in a study by Pavlov et al. confirmed the presence of 13 polioviruses, such as seven Sabin poliovirus type 1, four Sabin poliovirus type 3 and two Sabin poliovirus type 2 isolates $^{32}$. According to Oderinde et al., twenty cases with ten isolates each of poliovirus type 2 (Sabin-like) and circulating vaccine-derived poliovirus (cVDPV)-2 strains from the faeces of children with acute flaccid paralysis in Nigeria were identified and confirmed by $\mathrm{CDC}^{6}$.Baba et al. in their study, isolated and identified three wild poliovirus type, 11 Sabin type, combination of Sabin-types $1+2$ and 23 poliovirus, and 22 non-polio enteroviruses ${ }^{15}$.

In this study, no Wild-type poliovirus type 2 or 3 was found in this study. This owe to the fact that despite the severe challenges, a major milepost to poliovirus eradication has been the disappearance of indigenous wild poliovirus type 2 which was last found in West Africa in 1997, and last found anywhere in the world (in Uttar Pradesh, India) in 1999 ${ }^{33}$. Thus, in Nigeria, as in other parts of the world, the only current exposure to poliovirus type 2 is from use of the live, attenuated oral polio vaccine (OPV). Likewise 
in a study by Pavlov et $\mathrm{al}^{32}$, the majority of the polioviruses isolated from the stools of the immunodeficient children (10 of 13 isolates) were classified as 'oral poliovirus vaccine (OPV)-like viruses'. The finding in this study deviates from that of Grassly et $\mathrm{al}^{16}$, who reported that wild-type poliovirus serotypes 1 and 3 were isolated from the stool samples of $103(0.74 \%)$ and $104(0.74 \%)$ healthy contacts, respectively.

Also, in this study, the age distribution of children presenting with this paralysis shows that most cases occur between six months of age and four years of age. This shows that maternal antibodies (which normally wanes out after six months of age) prevents polio occurrence. This deviates from that of Grassly et $\mathrm{l}^{16}$. It was also observed that poliomyelitis occur in boys and girls but paralysis occurred more in boys as shown, 13 out of the 22 isolates $(59.1 \%)$ were from males while $9(40.9 \%)$ occurred in female children. This is consistent with previous studies which reported that poliovirus infection in children occurs equally in boys and girls but boys are more commonly paralyzed. However, the excreted Sabin poliovirus is capable of immunizing the unvaccinated children and promotes herd immunity.15Similarly, the excretion of combination of two polio serotypes indicates the child susceptibility to the missing serotype(s) and therefore indicates an immunity gap ${ }^{15}$.

As of 13th of April 2012, already 20 new cases of wild poliovirus have been reported in eight states compared to nine cases for the same time period in four states in $2011^{34}$. As of 22 June 2012, Nigeria has recorded 45 cases of wild poliovirus in 10 States compared to 25 cases in 6 States for the same period in $2011^{35}$. The distribution of poliovirus isolates in relation to locations showed highest number of the poliovirus isolates in Zamfara State, followed by Kaduna, Niger, and Sokoto States while the least were found in Abia and Delta States. According to the UNICEF 2012 updates, in key infected states like Borno, Kano, Sokoto and Yobe more than one in three children has received less than four doses of oral polio vaccine ${ }^{34-35}$. In line with the finding of this study, poliofree states like Kaduna and Niger were re-infected in $2012^{34-35}$. Nigeria contributes $90 \%$ to the polio burden in Africa and more than $50 \%$ of this year's cases worldwide are from Nigeria.35 However, no wild-type or Sabin-like poliovirus was isolated from Benue, Kebbi, Kogi, Kwara and Nasarawa States including Federal capital territory (FCT). This result is in consistent with the fact that
FCT is not an endemic State in Nigeria for wild poliovirus transmission ${ }^{11}$. According to the Rapid Assessment of Acute Flaccid Paralysis Surveillance (RAAFPS) conducted in Kebbi State in May 2011, Kebbi State has showed a steady decline in the number of AFP case reporting in the last 2 years from 234 cases in 2009 to 222 in $2010^{36}$. According to the same report, there were no polio-compatible cases reported in 2010 and all the 4 wild polioviruses reported in 2010 were orphan viruses ${ }^{36}$. However, the lack of wild poliovirus in these states may not necessarily reflect the true situation ${ }^{11}$.

Furthermore, children who were not vaccinated were found to harbor wild poliovirus serotypes 1 . This is consistent with the report of Adu et $\mathrm{al}^{25}$. Sabin poliovirus serotype 1 and 2 were found in children who had complete vaccination and Sabin poliovirus serotype 2 and mixtures of Sabin polio serotypes 1 and 2 as well as poliovirus 2 and poliovirus 3 was also found in children who had incomplete vaccination. This could be attributed to the fact that coverage by supplementary immunization with National Immunization Days (NIDs) has also been low and insufficient to block widespread wild poliovirus transmission in northern Nigeria $^{37-38}$. Low rates of oral polio vaccine coupled with poor sanitation, tropical conditions, crowding, and the prior eradication of the corresponding serotype of wild poliovirus are said to be the main risks factors for circulating vaccine derived poliovirus emergence ${ }^{25,39}$. However, we cannot be certain of the immune status of the children, as we could not measure serum immunoglobulin levels or neutralizing antibody titres to poliovirus, because only stool specimens, but not blood samples, are normally collected for investigation of suspected cases of acute flaccid paralysis ${ }^{23,25,40}$. According to Baba et al., the continued excretion of wild-type poliovirus among children above 5 years old vaccinated with oral polio vaccine contributes to the persistent circulation of these viruses in the environment and may limit the population immunity ${ }^{15}$.

\section{Conclusion}

This study further confirms the presence of Sabin and wild-type poliovirus among children in Nigeria. The isolation of Sabin strain of poliovirus is advantageous to the polio eradication program as it is capable of inducing natural immunity in susceptible hosts $^{11}$. Transmission of wild poliovirus among children with no vaccination and incomplete vaccinated children poses a serious threat to polio 
eradication program in Nigeria. Therefore, environmental and serological surveillance with larger sample size are important for monitoring poliovirus circulation in Nigeria. To prevent vaccine derived poliovirus (VDPV) emergence and spread, all countries should maintain high poliovirus vaccination coverage against all three poliovirus serotypes. Sensitive poliovirus surveillance to detect vaccine derived polioviruses will continue to increase in importance. Intensifying polio eradication strategies, all children $0-15$ years must be sufficiently immunized for high population immunity. In view of these, concerted effort should be made by the government and WHO towards complete eradication of poliomyelitis. Parents and guardians should be educated on the need to allow their children to receive the normal schedule of oral polio vaccine and booster doses given during national immunization programmes.

\section{Acknowledgement}

The authors would like to thank management and staff of World Health Organization National Reference Laboratory for Polio, Department of Virology, College of Medicine, and University of Ibadan for their support throughout the period of this study. The excellent technical assistance of Dr. Bola Oyemankinde, Miss Olufunke Adewale, Mr. Oluwaseun Adedeji among others are sincerely acknowledged.

\section{References}

1. Bãicu A, Combiescu M, Persu A, Oprioan G, Aubert-Combiescu A, Delpeyroux F. The Molecular Characterization Of Poliovirus Strains By The Rt-Pcr-Rflp Assay and Its Use In The Active Surveillance For Acute Flaccid Paralysis Cases In Romania Between 2001-2006. Romanian Archives Of Microbiology and Immunology, 2006; 65(3-4): 120-130

2. Sutter RW, Kew OM and Cochi SL. Poliovirus vaccine -live. In Plotkin SA and Orenstein WA (ed.), Vaccines, 4th ed. W.B. Saunders Company, Philadelphia, Pa; 2004; 651-705.

3. Kew OM, PF Wright, VI Agol, F Delpeyroux, H Shimizu, N Nathanson, and MA Pallansch. Circulating vaccine-derived polioviruses: current state of knowledge. Bulletin of World Health Orgnization, 2004; 82:16-23.

4. Yang CF, Chen HY, Jorba J, Sun HC, Yang SJ, Lee HC, Haung YC, Lin TY, Chen PJ, Shimizu
H, Nishimura Y, Utama A, Pallansch MA, Miyamura T, Kew OM, Yang JY. Intratypic recombination among lineages of type 1 vaccinederived poliovirus emerging during chronic infection of an immunodeficient patient. Journal of Virology, 2005; 79:12623-12634

5. Jenkins HE, Aylward RB, Gasasira A, Donnelly CA, Mwanza M, Corander J, Garnier S, Chauvin C, Abanida E, Pate MA, Adu FD, Baba MM, Grassly NC. Implications of a circulating vaccine-derived poliovirus in Nigeria. New England Journal of Medicine, 2010; 362:2360-2369.

6. Oderinde BS, Baba MM, Rimamnyang CM, Muhammad T, Ogunmola OO. Comparison of the Potency of Circulating Vaccine Derived Poliovirus Type 2 with Sabin like Type 2 by Micro Titration. Australian Journal of Basic and Applied Sciences, 2011; 5(11): 1352-1357

7. World Health Organization (WHO). Progress towards interruption of wild poliovirus transmission, January 2004 to March 2005. Weekly Epidemiological Records 2005; 80:149-155.

8. Minor PD and Almond JW. Poliovirus vaccines: molecular biology and immune response. In: Semler BL and Wimmer E (ed.), Molecular biology of picornaviruses. ASM Press, Washington, D.C. 2002; 381-390

9. Centre for Disease Control and Prevention (CDC). Laboratory surveillance for wild and vaccine-derived polioviruses - worldwide, January 2008-June 2009. Morbidity and Mortality Weekly Reports, 2009a; 58:950-954.

10. DeVries AS, J Harper, A Murray, C Lexau, L Bahta, J Christensen, E Cebelinski, S Fuller, S Kline, GS Wallace, JH Shaw, CC Burns, R Lynfield. Vaccine-Derived Poliomyelitis-12 Years after Infection in Minnesota. New England Journal of Medicine 2011;364:2316-2323.

11. Oderinde BS, Baba MM, Barde AD and Dawurung J. Polioviruses in Apparently Healthy Secondary School Students in FCT Abuja, Nigeria. Archives of Applied Science Research, 2012; 4 (3):1432-1435

12. De Jesus NH. Epidemics to eradication: the modern history of poliomyelitis. Virology Journal, 2007;4:70.

13. Centre for Disease Control and Prevention (CDC). Progress toward interruption of wild poliovirus transmission - worldwide, 2008. Morbidity and Mortality Weekly Reports, 2009b; 58:308-312. 
14. Centre for Disease Control and Prevention (CDC). Paralytic poliomyelitis \pm United States,

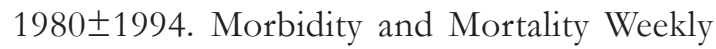
Reports, 1997; 46, 79-83.

15. Baba MM, Oderinde BS, Patrick PZ, Jarmai MM. Sabin and wild polioviruses from apparently healthy primary school children in northeastern Nigeria. Journal of Medical Virology, 2012; 84:358364

16. Grassly NC, Jafari H, Bahl S, Durrani S, Wenger J, Sutter RW, Aylward RB. Asymptomatic WildType Poliovirus Infection in India among Children with Previous Oral Poliovirus Vaccination. Journal of Infectious Diseases, 2010; 201(10):1535-1543.

17. Centre for Disease Control and Prevention (CDC). Update on vaccine-derived poliovirusesworldwide, January 2008-June 2009. Morbidity and Mortality Weekly Reports, 2009c; 58:1002-1006.

18. Centre for Disease Control and Prevention (CDC). Tracking progress toward global polio eradication-worldwide, 2009-2010. Morbidity and Mortality Weekly Reports, 2011a;60:441-445.

19. Wassilak S, Pate MA, Wannemuehler K, et al. Outbreak of type 2 vaccine-derived poliovirus in Nigeria, 2005-2009: emergence and widespread circulation in an underimmunized population. Journal of Infectious Diseases, 2011;203: 898-909.

20. Centre for Disease Control and Prevention (CDC). Update on Vaccine-Derived Polioviruses - Worldwide, July 2009-March 2011. Morbidity and Mortality Weekly Reports, 2011b. http:// periodicals.faqs.org/201107/2400110511.html

21. Tebbens RJD, Pallansch MA, Kew OM, C'aceres VM, Jafari H, Cochi SL, Sutter RW, Aylward $\mathrm{RB}$ and Thompson KM. Risks of Paralytic Disease Due toWild or Vaccine-Derived Poliovirus After Eradication. Risk Analysis, 2006; 26(6): 1471-1505

22. World Health Organization (WHO). Polio laboratory manual 4th edition. Department of Immunization, Vaccines and Biologicals $\mathrm{CH}$ 1211, World Health Organization, Geneva 27, Switzerland, 2004a; WHO/IVB/04.10, pp1167

23. World Health Organization (WHO). Laboratory surveillance for wild and vaccine-derived polioviruses, January 2003-June 2004. Weekly Epidemiological Records, 2004b; 79: 393-398

24. World Health Organization (WHO). Supplemental Polio Laboratory Manual on New
Algorithm. Polio Lab Network Quarterly Update, December 2006, Volume XIII, Issue 4, pages 1-4, WHO Global Polio Eradication Initiatives, Geneva, Switzerland.

25. Adu FD, Iber J, Bukbuk D, Gumede N, Yang S, Jorba J, Sule WF, Yang C, Burns C, Pallansch M, Harry T, and Kew O. Isolation of recombinant type 2 vaccine-derived poliovirus (VDPV) from a Nigerian child. Virus Research, 2007; 127:1725

26. Oberste MS, Nix WA, Maher K and Pallansch MA. Improved molecular Identification of enteroviruses by RT-PCR and amplicon sequencing. Journal of Clinical Virology, 2003; 26: 375-377

27. Nix WA, Oberste MS and Pallansch MA. Sensitive, seminested PCR amplification of VPI sequences for direct identification of all enterovirus serotypes from original clinical specimens. Journal of Clinical Microbiology, 2006; 44(8); 001-007.

28. ERC. The 23rd Meeting of the Expert Review Committee (ERC) on Polio Eradication Initiative \& Routine Immunization, Abuja, Nigeria, 2829 March 2012, pages 1-6

29. Centers for Disease Control and Prevention (CDC). Laboratory surveillance for wild polio virus and vaccine-derived poliovirus, 2000-2001. Morbidity and Mortality Weekly Reports, 2002; 51:369-371.

30. Centers for Disease Control and Prevention (CDC). Laboratory surveillance for wild and vaccine-derived polioviruses, January 2002June 2003. Morbidity and Mortality Weekly Reports, 2003; 52:913-916.

31. Centre for Disease Control and Prevention (CDC). Progress towards interruption of wild poliovirus transmission-worldwide, January 2005-March 2006. Morbidity and Mortality Weekly Report (MMWR), 2006; 55:458-462

32. Pavlov DN, Van Zyl WB, Van Heerden J, Kruger M, Blignaut L, Grabow WOK, and Ehlers MM. Prevalence of vaccine-derived polioviruses in stools of immunodeficient children in South Africa. Journal of Applied Microbiology, 2006; 101 (6):1367-1379

33. World Health Organization (WHO). Transmission of wild poliovirus type 2-apparent global interruption. Weekly Epidemiological Records, 2001; 76: 95-97 
34. United Nations Children's Fund (UNICEF). A worrisome upward trend in Nigeria [Internet]. UNICEF Monthly Polio Situation Update in Nigeria, 2012 April 13, 2012.

35. United Nations Children's Fund (UNICEF). Polio Situation in Nigeria [Internet]. UNICEF Monthly Polio Situation Update in Nigeria, 2012 June 22, 2012. [cited 2012 July 18]

36. RAAFPS. Rapid Assessment of Acute Flaccid Paralysis (AFP) Surveillance in Kebbi State, Nigeria, Conducted during May 16 to 21, 2011, pp1-9

37. Centre for Disease Control and Prevention (CDC). Laboratory surveillance for wild and vaccine-derived polioviruses, January 2004-June 2005. Morbidity and Mortality Weekly Report 2005; 54:958-961
38. Centre for Disease Control and Prevention (CDC). Progress towards poliomyelitis eradication-Nigeria, 2005-2006. Morbidity and Mortality Weekly Report 2007; 56:278-281

39. Kew OM, Sutter RW, de Gourville EM, Dowdle WR, Pallansch MA. Vaccine-derived polioviruses and the endgame strategy for global polio eradication. Annual Review of Microbiology, 2005; 59: 587-635

40. World Health Organization (WHO). Field Guide for supplementary activities aimed at achieving polio eradication, 1996 revision, WHO/EPI/ GEN/95.01 Rev.1, World Health Organization, Geneva, Switzerland, 1997; pp153. 